\title{
Spatial distribution of crack structure in the focal area of a volcanic earthquake swarm at the Hakone volcano, Japan
}

\author{
Yu Nihara ${ }^{1}$, Keiichi Tadokoro ${ }^{1}$, Yohei Yukutake ${ }^{2}$, Ryou Honda $^{2}$, and Hiroshi Ito ${ }^{2}$ \\ ${ }^{1}$ Graduate School of Environmental Studies, Nagoya University, Nagoya, Japan \\ ${ }^{2}$ Hot Springs Research Institute of Kanagawa Prefecture, Odawara, Japan \\ (Received February 16, 2012; Revised May 1, 2012; Accepted May 10, 2012; Online published February 19, 2013)
}

\begin{abstract}
We have performed shear wave splitting analyses for seismograms recorded at stations located just above, and outside, the focal area of the earthquake swarm at the Hakone volcano, Japan, in August 2009. Average values of the direction of faster split shear wave polarization $(\Phi)$ at two stations above the focal area correspond to each focal alignment of the earthquake swarm. In contrast, average values of $\Phi$ at three stations outside the focal area correspond to the direction of the maximum horizontal compressional stress. We found that the average values of the time lag between the two split shear waves inside the focal area are relatively high compared with those outside the focal area. These facts suggest that cracks with a high density aligned parallel to the faults of the earthquake swarm in the focal area. Crustal fluid was selectively injected into this pre-existing cracked media accompanied by effective normal stress reduction in the cracks, resulting in the earthquake swarm.
\end{abstract}

Key words: Shear wave splitting, Hakone volcano, crack structure, earthquake swarm.

\section{Introduction}

It is generally believed that earthquake swarms are caused by the migration of crustal fluid (e.g., Nur, 1974). Earthquake swarms sometimes occur in geothermal, or volcanic, areas, e.g., in the Long Valley Caldera of California (Savage and Cockerham, 1984) and the Yellowstone volcanic field of the western United States (Farrell et al., 2009). Hill (1977) proposed a model in which volcanic earthquake swarms are caused by faults arranged according to the local stress field, with magmatic/fluid intrusions in brittle rock. Sibson (1996) suggested a model of swarm activity in which earthquakes are triggered by fluid migration through a highly permeable structure. It is therefore important to investigate crack distribution related to the swarm to understand its occurrence in volcanic areas.

Many intense earthquake swarms have been reported in the Hakone caldera, Japan. There were major swarms in 2001, 2006, and 2009 (Tanada et al., 2002, 2007; Yukutake et al., 2011). Mannen (2008) put forth a model in which increased pore pressure in cracks, caused by hydrothermal injection, generated the Hakone volcano swarms. The 2009 swarm activity was from 4 to 12 August. Yukutake et al. (2011) located the hypocenters of 1156 events during this period with the Double-Difference method. Hypocenters of the 2009 swarm were distributed on two vertical planes. The activity migrated from the depths, and the north, toward shallow zones and the south. This hypocenter migration also suggests a relationship between the swarm and crustal fluid (Yukutake et al., 2011).

Copyright (c) The Society of Geomagnetism and Earth, Planetary and Space Sciences (SGEPSS); The Seismological Society of Japan; The Volcanological Society of Japan; The Geodetic Society of Japan; The Japanese Society for Planetary Sciences; TERRAPUB.

doi:10.5047/eps.2012.05.005
Shear wave splitting is a phenomenon in which an $S$ wave propagating through anisotropic media splits into two quasi $S$-waves. Each quasi $S$-wave polarizes into orthogonal directions with different velocities. In the case of shear wave splitting caused by the preferred orientation of cracks, the quasi $S$-wave polarized parallel to crack orientation travels faster than the other quasi $S$-wave. The orientation of faster $S$-wave polarization is therefore parallel to the direction of maximum horizontal compressional stress (e.g., Crampin, 1987; Kaneshima et al., 1987). In contrast, the anisotropic behavior near faults is different from that around the fault just after the earthquake occurrence. The orientation of faster $S$-wave polarization is parallel to the fault strike (e.g., Zhang and Schwartz, 1994). Tadokoro et al. (1999) concluded that the fault-parallel direction of faster $S$-wave polarization is caused by new fractures generated by shear faulting during the mainshock. Shear-wave splitting analysis is the favored method to detect local seismic structure, as well as to estimate the direction of the tectonic stress field.

We performed shear-wave splitting analysis for seismograms recorded at stations located just above and outside the focal area of the earthquake swarm at Hakone volcano in August 2009, to depict crack distribution and address the relationship between crack structure and swarm occurrence.

\section{Data and Method}

We used seismograms of earthquakes recorded at four permanent stations (KZR, KIN, KZY, and KOM) and one temporary station (T.OSS) in the Hakone caldera, during the period June 2009 through June 2010 (Fig. 1). The four permanent stations are operated by the Hot Springs Research Institute (HSRI) of Kanagawa Prefecture, Japan. The stations KZR and T.OSS are just above the focal area of 
(a)

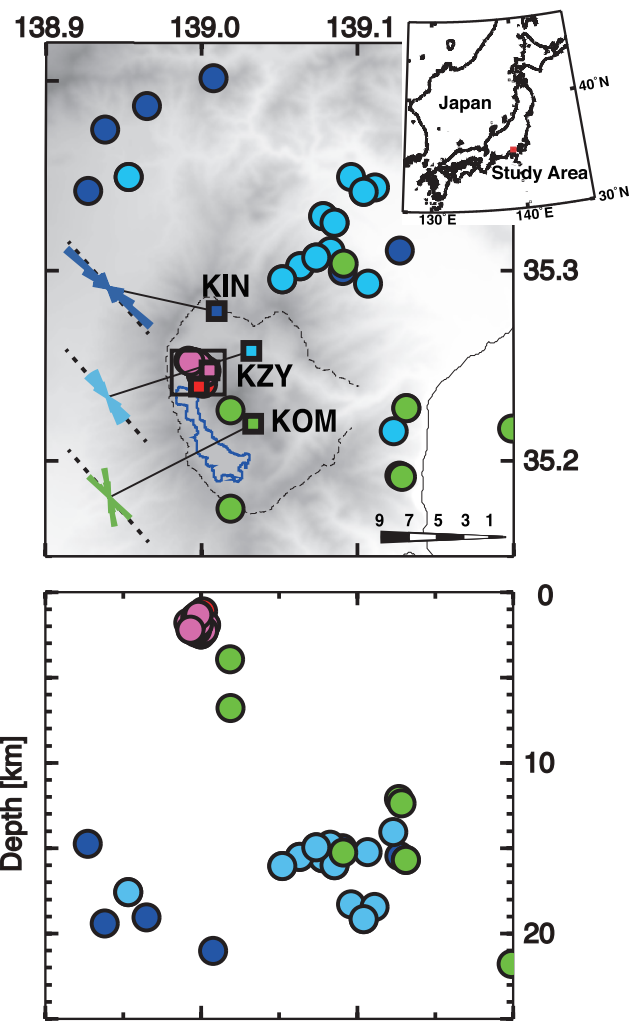

(b)

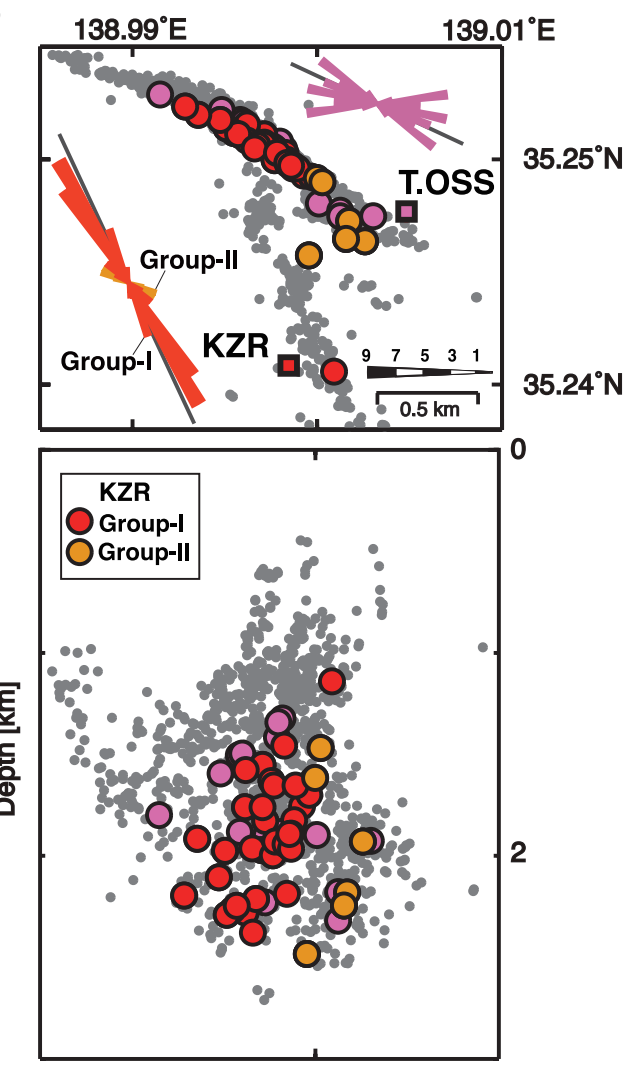

Fig. 1. Maps showing distribution of seismic stations (squares) and hypocenters (solid colored circles). Earthquakes used for analysis at each station are depicted by different colors for each station. Rose diagrams show $\Phi$ and the number of data at each station. Values between $0^{\circ}$ and $180^{\circ}$ are plotted again between $180^{\circ}$ and $360^{\circ}$, because the value of $\Phi$ is a nonvector direction. Solid lines indicate the fault strike beneath each station (Yukutake $e t$ al., 2011). Dotted lines show the orientation of the maximum horizontal compressional stress in this region, estimated from a stress inversion analysis (Yukutake et al., 2007). (a) Dashed line indicates outer rim of the Hakone caldera. The rectangle shows the area of Fig. 1(b). (b) Red and orange solid circles indicate Group-I and II events used in the analysis at KZR (see text). Gray dots indicate hypocenters of other earthquakes during the analysis period.

Table 1. The number of data and results of $S$-wave splitting analysis.

\begin{tabular}{ccccc}
\hline Station & & Number of events & $\Phi\left(\mathrm{N}^{\circ} \mathrm{E}\right)$ & $\mathrm{DT}(\mathrm{ms})$ \\
\hline KZR & (Total) & 40 & $143 \pm 3$ & $86 \pm 2$ \\
& $($ Group-I $)$ & 34 & $148 \pm 2$ & $86 \pm 3$ \\
& (Group-II) & 6 & $113 \pm 2$ & $86 \pm 2$ \\
T.OSS & 36 & $105 \pm 3$ & $49 \pm 4$ \\
KIN & 13 & $130 \pm 4$ & $43 \pm 7$ \\
KZY & 13 & $143 \pm 3$ & $52 \pm 5$ \\
KOM & 7 & $156 \pm 7$ & $54 \pm 12$ \\
\hline
\end{tabular}

the 2009 swarm activity. At three permanent stations (KZR, $\mathrm{KZY}$, and KOM), three-component velocity seismometers are installed in boreholes. Seismometers are installed on the surface at T.OSS and KIN. The seismometer natural frequency is $1 \mathrm{~Hz}$, except for T.OSS $(2 \mathrm{~Hz})$. The sampling rate is $200 \mathrm{~Hz}$ at KZR, KOM and T.OSS, and $120 \mathrm{~Hz}$ at KIN and KZY.

Figure 1 shows stations and events in the present analysis. We used events with incident angles less than $35^{\circ}$, to avoid the $S$ - $P$ converted wave effect (Booth and Crampin, 1985). We calculated the incident angle from a coordinate of events and stations, assuming that paths from the focus to the station are straight lines. We also selected seismograms of events with a magnitude greater than 1.0, and clear $S$ wave first motion. We adopted the result of hypocenter determination through the Double-Difference method by Yukutake et al. (2011) as the hypocenter catalog for the period of 4-12 August, and the catalog produced by HSRI for the other term. Since the purpose of this study is to find the relation between earthquake swarm occurrence and crack distribution, we used only local events in the focal area of the swarm for analysis at the stations just above that area (KZR and T.OSS). The focal depths used are shallower than $2.5 \mathrm{~km}$ at the stations KZR and T.OSS; event depths for KIN, KZY and KOM are 3-25 km. The numbers of selected events are listed in Table 1.

Figure 2 shows an example of waveforms with shearwave splitting, recorded at KZR. We used a method that computes the cross-correlation coefficient with the rotation of coordinate axes (e.g., Shih and Meyer, 1990), with 
(a)
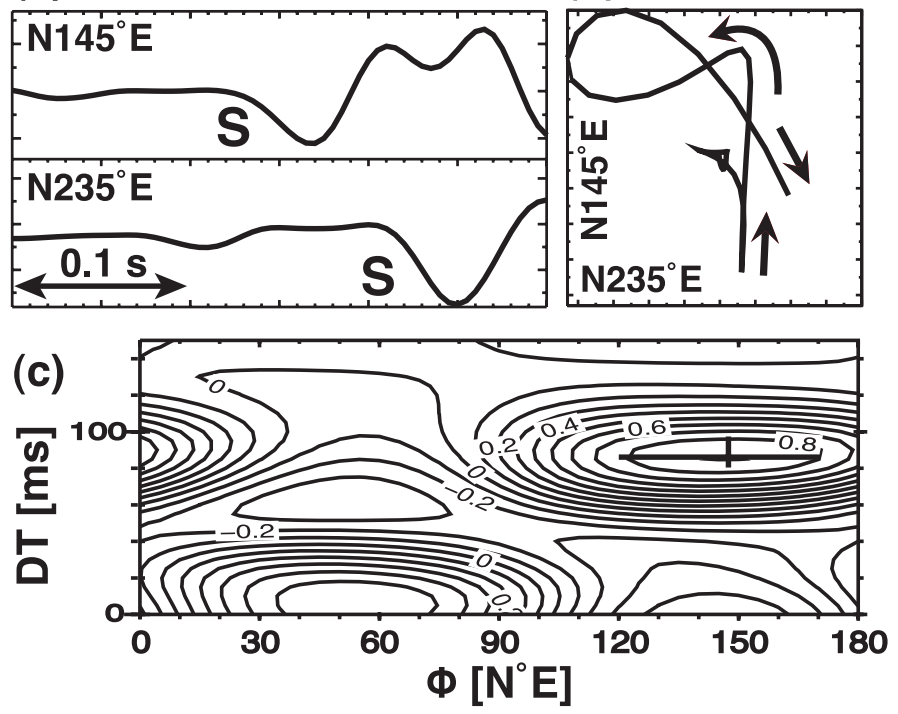

Fig. 2. (a) Example of horizontal component seismograms with shear-wave splitting, recorded at station KZR. Seismograms are rotated to the leading/lagging coordinate system, i.e., one axis is rotated to the direction at which the cross-correlation coefficient is maximized. Two quasi $S$-phases in the seismograms have similar shapes and shift by $85 \mathrm{~ms}$, the computed value of $D T$. (b) Particle motion plot containing $S$-wave first motion shown in Fig. 2(a). Initial polarization is $\mathrm{N} 145^{\circ} \mathrm{E}$, the computed value of $\Phi$, and particle motion changes abruptly to the orientation $\mathrm{N} 235^{\circ} \mathrm{E}$ at the arrival time of the lagging $S$-wave. (c) Contour plot of cross-correlation coefficient. Plus shows $95 \%$ confidence region.

steps of $5^{\circ}$ and shifting the time of one component waveform by steps of $5 \mathrm{~ms}$ for the two horizontal component waveforms with low-pass filtering at $10 \mathrm{~Hz}$. The width of the analysis time window is 0.3 seconds from the $S$ wave first motion. We determined the rotated angle and lag time as the direction of faster split shear-wave polarization $(\Phi)$ and lag between the two split shear waves $(D T)$, respectively, when the cross-correlation coefficient was maximized. Figure 2(c) shows an example of grid maps of $(\Phi$, $D T$ ) for the cross-correlation coefficient. We quantified the error in the solution based on the reliability of the estimation of the correlation coefficient with Fisher's z-transformation. We omitted events with a confidence interval wider than $20 \mathrm{~ms}$ (about $1 / 4$ wavelength), as they were considered to have unreliable data. We also omitted events in which the lower limit of the confidence interval was negative, because $D T$ is defined as a positive value.

\section{Results and Discussion}

The average and standard error of $\Phi$ at stations just above the focal area (KZR and T.OSS) are shown in Table 1 and Fig. 1. The angle $\Phi$ is measured clockwise from the north. Since we used almost the same events at KZR and T.OSS, the $\Phi$ difference at the two stations obviously reflects a difference of anisotropy in the paths. Yukutake et al. (2011) detected focal alignment by the Double-Difference method and concluded that the alignments correspond to small faults. Directions of the northern and southern focal alignments were $\mathrm{N} 110^{\circ} \mathrm{E}$ and $\mathrm{N} 155^{\circ} \mathrm{E}$, respectively. Average $\Phi$ at T.OSS $\left(105 \pm 3^{\circ}\right)$ was almost parallel to the direction of the northern focal alignment $\left(\mathrm{N} 110^{\circ} \mathrm{E}\right)$.

In addition, we found a variation of $\Phi$ with azimuthal angle at KZR. We divide the events of the KZR analysis into the following two groups: (1) events belonging to the focal alignment just below KZR, with an azimuth parallel to the focal alignment below this station $\left(\mathrm{N} 22^{\circ} \mathrm{W}-\mathrm{N} 8^{\circ} \mathrm{E}\right)$ (Group-I); and (2) other events (Group-II) (Fig. 1(b)). Averages of $\Phi$ for Groups I and II are $148 \pm 2^{\circ}$ and $113 \pm 2^{\circ}$, respectively; these values are significantly different. Because the observed shear-wave splitting reflects anisotropy of the ray path, the difference of $\Phi$ between Group-I and Group-II shows a different origin of anisotropy on each ray path. Most of the ray paths from Group-I pass through the focal region beneath KZR. Thus, results from GroupI events indicate anisotropy in the region, along with the focal alignment. Group-II manifests anisotropy outside the focal region (the region between the northern focal alignment and KZR). The average $\Phi$ for Group-I $\left(148 \pm 2^{\circ}\right)$ was almost parallel to the direction of southern focal alignment $\left(\mathrm{N} 155^{\circ} \mathrm{E}\right)$. The fact that $\Phi$ averages at T.OSS and for Group-I at KZR nearly parallel the direction of each focal alignment beneath the stations suggests that cracks aligned parallel to the faults of the earthquake swarm were distributed inside the swarm focal region. The crack structure indicates a fine structure in the small faults reported by Yukutake et al. (2011).

Averages, and a standard error of $\Phi$, at three stations outside the focal area are also shown in Table 1 and Fig. 1. The $\Phi$ averages at KIN and KZY parallel to the direction of the maximum horizontal compressional stress $\left(\mathrm{N} 139^{\circ} \mathrm{E}\right)$ in the study area (Yukutake et al., 2007). The rose diagram at KOM in Fig. 1 has two peaks. One of the two peaks is at $135^{\circ}$, parallel to the direction of maximum horizontal compressional stress. This parallelism indicates that cracks generated by the tectonic stress field prevailed outside the focal area of the swarm. Honda and Tanada (2006) also reported several peaks of the $\Phi$ direction at KOM from analysis for the period 2002 and 2005, similar to the present result. It is believed that the sparse distribution of $\Phi$ reflects the universal crack distribution just below KOM. 


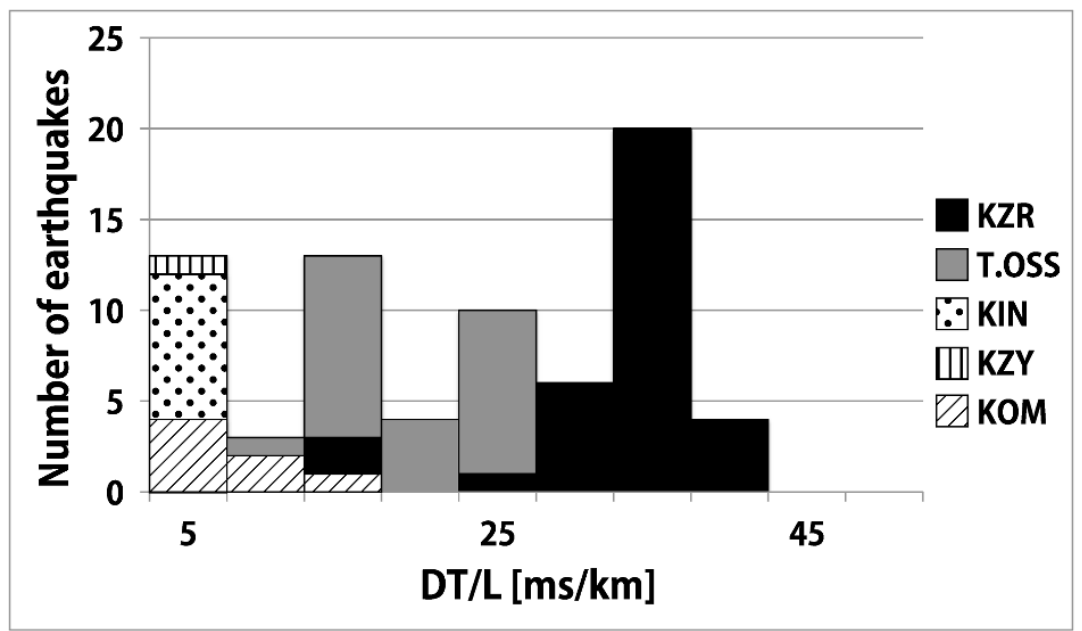

Fig. 3. Histogram of $D T$ normalized by path length in the anisotropic region, $L$.

Averages, and standard errors, of $D T$ at the stations on the earthquake swarms are $86 \pm 2 \mathrm{~ms}$ at KZR, and $49 \pm 4 \mathrm{~ms}$ at T.OSS (Table 1). In contrast, $D T$ averages at the stations outside the swarm are $43 \pm 7 \mathrm{~ms}, 52 \pm 5 \mathrm{~ms}$ and $54 \pm 12$ ms at KIN, KZY, and KOM, respectively (Table 1). Figure 3 shows histograms of $D T / L$, the value of $D T$ divided by the length of ray path in the anisotropic region $L$. The $D T / L$ at KZR is calculated only from the result of Group-I events. The lower limit of the observed anisotropy regions was not clearly identified from our measurement. We calculated $D T / L$ assuming that $L$ is the length of the entire path. As a result, the values at KZR and T.OSS in Fig. 3 are minima of $D T / L$. Leary et al. (1990) mentioned that there is stress-induced anisotropy throughout the brittle crust in tectonically active areas like our study area. Stress-induced anisotropy was observed at various depths in the upper crust (e.g., Kaneshima and Ando, 1989). We thus calculated values of $D T / L$ at KIN, KZY and KOM, where the stressinduced anisotropy was observed, assuming that the entire path is within the anisotropic region. The $D T / L$ reflects the degree of anisotropy, i.e., crack density, beneath the station. Values of $D T / L$ at KZR and T.OSS, which are just above the swarm focal area, are greater than those at the stations outside the focal area. In particular, the value at KZR is significantly higher than those at other stations. Therefore, the crack density inside the focal area is significantly greater than that outside it.

The degree of anisotropy $k$ is defined as

$$
k=\frac{\Delta V_{S}}{V_{S}} \approx \frac{V_{S} D T}{L},
$$

where $\Delta V_{S}$ is the velocity difference between the two split shear waves, and $V_{S}$ is the $S$-wave velocity in the isotropic medium (Hudson, 1981). We calculated averages of $k$ at each station, by substituting $D T / L$ and $V_{S}$ calculated from the velocity structure of Yukutake et al. (2010) into Eq. (1). The $k$ averages inside the swarm area are $5.9 \%$ and $3.5 \%$ at KZR and T.OSS, respectively. These values are much larger than those outside the swarm area $(0.5 \%, 0.8 \%$, and $1.5 \%$ at KIN, KZY and KOM, respectively). The high $k$ averages at KZR and T.OSS indicate that the highly-fractured medium existed just below KZR and T.OSS. Roberts and Crampin (1986) also reported a highly-fractured medium shallower than $2 \mathrm{~km}$ at a geothermal area near Cornwall, United Kingdom.

Yukutake et al. (2011) concluded that the 2009 swarm activity was caused by water migration in the source region. The highly-fractured medium can be the cause of the crustal fluid migration into the source region: the preexisting cracks whose orientations are parallel to the epicenter alignment with high density caused the crustal fluid to inject selectively into the cracked media, resulting in pore pressure increase (normal stress reduction) and the 2009 swarm. Daniel et al. (2011) reported that effective stress decreased nearly $8 \mathrm{MPa}$ under constant shear stressing-rate conditions caused by pore pressure increase, during the 2003-2004 Ubaye seismic swarm activity in France. Nodal planes of the focal mechanism revealed by Yukutake et al. (2011) are parallel to strikes of the northern and southern focal alignments. These findings support our idea.

\section{Conclusion}

We found a spatial variation of crack orientation and density related to the 2009 earthquake swarm at Hakone volcano. Crack orientations inside the focal area were parallel to each fault strike of the swarm. In contrast, the orientation outside the focal area corresponded with the direction of the original maximum horizontal compressional stress. Crack density inside the focal area is extremely great, 3.5-5.9\%. We conclude that crustal fluid selectively injected into preexisting cracked media of high crack density, accompanied by effective normal stress reduction in the cracks, resulting in the earthquake swarm. $S$-wave splitting analysis in the focal area of a volcanic earthquake swarm is a useful means to investigate the relation between crack distribution and swarm occurrence.

Acknowledgments. The comments from Professor Martha Kane Savage and an anonymous reviewer are instructive in improving our manuscript. We used the Generic Mapping Tool (Wessel and Smith, 1995) to draw the maps. This research was partly supported by grants-in-aid from the Ministry of Education, Culture, Sports, Science and Technology, Japan (No. 21740328). 


\section{References}

Booth, D. C. and S. Crampin, Shear-wave polarizations on a curved wavefront at an isotropic free-surface, Geophys. J. R. Astron. Soc., 83, 31-45, 1985.

Crampin, S., The geological and industrial implications of extensivedilatancy anisotropy, Nature, 328, 491-496, 1987.

Daniel, G., E. Prono, F. Renard, F. Thouvenot, S. Hainzl, D. Marsan, A. Helmstetter, P. Traversa, J. L. Got, L. Jenatton, and R. Guiguet, Changes in effective stress during the 2003-2004 Ubaye seismic swarm, France, J. Geophys. Res., 116, B01309, doi:10.1029/2010JB007551, 2011.

Farrell, J., S. Husen, and R. B. Smith, Earthquake swarm and b-value characterization of the Yellowstone volcano-tectonic system, J. Volcanol. Geotherm. Res., 188, 260-276, doi:10.1016/j.jvolgeores.2009.08.008, 2009.

Hill, D. P., A model for earthquake swarms, J. Geophys. Res., 82, 13471352, 1977.

Honda, R. and T. Tanada, Variations of S-wave anisotropy in space and time in the crust of western Kanagawa, Japan, Bull. Hot Springs Res. Inst. Kanagawa Pref., 38, 47-52, 2006 (in Japanese with English abstract).

Hudson, J. A., Wave speeds and attenuation of elastic waves in material containing cracks, J. Geophys. R. Astron. Soc., 64, 133-150, 1981.

Kaneshima, S. and M. Ando, An analysis of split shear waves observed above crustal and uppermost mantle earthquakes beneath Shikoku, Japan: Implications in effective depth extent of seismic anisotropy, $J$. Geophys. Res., 94, 14077-14092, 1989.

Kaneshima, S., M. Ando, and S. Crampin, Shear-wave splitting above small earthquakes in the Kinki district of Japan, Phys. Earth Planet. Inter., 45, 45-58, 1987.

Leary, P. C., S. Crampin, and T. V. McEvilly, Seismic fracture anisotropy in the Earth's crust: An overview, J. Geophys. Res., 95, 11105-11114, 1990.

Mannen, K., Hakone caldera: structure, model of formation, and role in presentday volcanism, Res. Rep. Kanagawa Pref. Mus. Nat. Hist., 13, 61-76, 2008 (in Japanese with English abstract).

Nur, A., Matsushiro, Japan, earthquake swarm: confirmation of the dilatancy-fluid diffusion model, Geology, 2, 217-221, 1974.

Roberts, G. and S. Crampin, Shear-wave polarizations in a Hot-DryRock geothermal reservoir: Anisotropic effects of fractures, Int. J. Rock. Mech. Min. Sci., 23, 291-302, 1986.
Savage, J. C. and R. S. Cockerham, Earthquake swarm in Long Valley Caldera, California, January 1983: Evidence for dike inflation, J. Geophys. Res., 89, 8315-8324, doi:10.1029/JB089iB10p08315, 1984.

Shih, X. R. and R. P. Meyer, Observation of shear wave splitting from natural events: South Moat of Long Valley Caldera, California, June 29 to August 12, 1982, J. Geophys. Res., 95, 11179-11195, 1990.

Sibson, R. H., Structural permeability of fluid-driven fault-fracture meshes, J. Struct. Geol., 18, 1031-1042, doi:10.1016/0191-8141(96)00032-6, 1996.

Tadokoro, K., M. Ando, and Y. Umeda, S wave splitting in the aftershock region of the 1995 Hyogo-ken Nanbu earthquake, J. Geophys. Res., 104, 981-992, 1999.

Tanada, K., Y. Daita, H. Ito, and K. Hakamada, Swarm activity of Hakone volcano in 2001, Bull. Hot Springs Res. Inst. Kanagawa Pref. Catfish Lett., 52, 1-4, 2002 (in Japanese).

Tanada, K., R. Honda, M. Harada, Y. Yukutake, and H. Ito, Seismic activity in the Kanagawa prefecture in 2006, Bull. Hot Springs Res. Inst. Kanagawa Pref. Catfish Lett., 57, 1-13, 2007 (in Japanese).

Wessel, P. and W. H. F. Smith, New version of the generic mapping tools released, Eos Trans. AGU, 76(33), 329, doi:10.1029/95EO00198, 1995.

Yukutake, Y., T. Tanada, R. Honda, M. Harada, H. Ito, K. Shiomi, and K. Obara, The spatial distribution of focal mechanisms in and around the region of western Kanagawa Prefecture, Japan Geoscience Union Meeting, Chiba, Japan, 2007.

Yukutake, Y., T. Tanada, R. Honda, M. Harada, H. Ito, and A. Yoshida, Fine fault structures in the geothermal region of Hakone volcano, revealed by well-resolved earthquake hypocenters and focal mechanisms, Tectonophysics, 489, 104-118, doi:10.1016/j.tecto.2010.04.012, 2010.

Yukutake, Y., H. Ito, R. Honda, M. Harada, T. Tanada, and A. Yoshida, Fluid-induced swarm earthquake sequence revealed by precisely determined hypocenters and focal mechanisms in the 2009 activity at Hakone volcano, Japan, J. Geophys. Res., 116, B04308, doi:10.1029/2010JB008036, 2011.

Zhang, Z. Z. and S. Y. Schwartz, Seismic anisotropy in the shallow crust of the Loma Prieta segment of the San Andreas fault system, J. Geophys. Res., 99, 9651-9661, 1994.

Y. Nihara (e-mail: nihara@seis.nagoya-u.ac.jp), K. Tadokoro, Y. Yukutake, R. Honda, and H. Ito 